\title{
Detecting Magnetic Resonance through Quantum Jumps of Single Molecules
}

\author{
A. C. J. Brouwer, E. J. J. Groenen, and J. Schmidt \\ Centre for the Study of Excited States of Molecules, Huygens Laboratory, University of Leiden, 2300 RA Leiden, The Netherlands
} (Received 8 December 1997)

\begin{abstract}
The fluorescence of a single molecule exhibits light and dark periods which reveal the jumping back and forth between the singlet and triplet manifold. In this paper we demonstrate how microwaveinduced changes to the distribution of the triplet-residence times can be used to detect magneticresonance transitions between triplet sublevels and to determine their kinetics. By synchronizing resonant microwave pulses with the quantum jumps of a terrylene molecule it proved possible to record transient-nutation signals with a high contrast. [S0031-9007(98)05942-0]
\end{abstract}

PACS numbers: $39.30 .+\mathrm{w}, 33.20 . \mathrm{Bx}, 33.50 .-\mathrm{j}, 76.70 . \mathrm{Hb}$

In recent years, optical spectroscopy of single molecules has allowed a wide range of experiments on an increasing number of molecular systems [1]. Still, magnetic-resonance experiments involving a paramagnetic state were as yet demonstrated only for single pentacene molecules [2,3] and individual $N-V$ centers in diamond [4]. Since the signal detected in single-molecule experiments usually consists of the fluorescence emitted by the molecule upon laser excitation, systems apt for single-molecule spectroscopy should have a high fluorescence rate. The fluorescence concerns a transition between the singlet ground state $\left(S_{0}\right)$ and the lowest excited singlet state $\left(S_{1}\right)$. The lowest triplet state $T_{1}$ (where two unpaired electrons combine to form a spin $S=1$ ) lies below $S_{1}$ and becomes occupied, with a low degree of probability, from the $S_{1}$ state through a spin-forbidden process called intersystem crossing (ISC). The $T_{1}$ state is relatively long lived. While present in $T_{1}$, the molecule does not participate in excitation/emission cycles and thus ceases to fluoresce. For this reason molecules with a low ISC, which visit the triplet state infrequently, are favored in single-molecule spectroscopy. However, this very property makes it hard to perform optically detected magnetic-resonance (ODMR) spectroscopy on the triplet state of a single molecule. In such an ODMR experiment the resonant absorption of microwaves connecting two triplet sublevels is detected through a change of the fluorescence rate. The fluorescence rate may change when two triplet sublevels with different lifetimes are coupled, causing a change in the average triplet lifetime.

Instead of detecting the average fluorescence, our method involves the detection and analysis of the length of the dark periods that reflect the presence of the molecule in the triplet state. Such an experiment was performed on terrylene embedded at low concentration in a $p$-terphenyl host crystal (Fig. 1). Terrylene is known to have a quantum yield for ISC from $S_{1}$ to $T_{1}$ as low as $10^{-5}$ [5] and as yet it proved impossible to observe the magnetic-resonance transitions in its $T_{1}$ state. We detected single terrylene molecules by cooling a crystal to $1.8 \mathrm{~K}$ and spectrally selecting molecules using a single-mode $\mathrm{cw}$ dye laser. Molecule 1 was selected at
$17286.08 \mathrm{~cm}^{-1}$, in the red wing of the origin of the $X_{2}$ site [5]. Owing to the short fluorescence lifetime of terrylene (3.8 ns [6]) and the low ISC, the number of detected photons is so high that it is possible to see the molecule jump back and forth between the singlet and triplet manifold [7]. As seen in Fig. 2, the molecule goes dark and then lights up. The length of a dark period reveals the residence time in the triplet state. By building a histogram of triplet-residence times (Fig. 2), a statistic of the decay of the triplet state can be constructed [8]. Since there are three triplet sublevels, a three-exponential decay is expected. The histogram seems to be roughly biexponential which is consistent with the fact that planar aromatic hydrocarbons typically have two short-lived triplet sublevels with similar lifetimes and one long-lived triplet sublevel [9]. Note that the shape of the histogram should change when a short-lived level is coupled to the long-lived level by way of microwaves. This provides an opportunity for detecting a magnetic-resonance transition even when the triplet is visited infrequently and even when the relative yields and lifetimes of the sublevels are such that the average triplet-residence time hardly changes, making conventional ODMR inapplicable.

To make quantum-jump-detected magnetic resonance practical, the fluorescence signal must be processed in real time. An efficient software filter was implemented for this purpose. It runs over the fluorescence photon-counting signal read via a multichannel scaler and determines the jump times by referencing an external time base. The resultant "signal" is buffered for use in a variety of experiments and can be correlated to parameters (such as the microwave frequency) that are also synchronized with the time base. The top of Fig. 3 shows a 2D histogram constructed by counting the triplet-residence

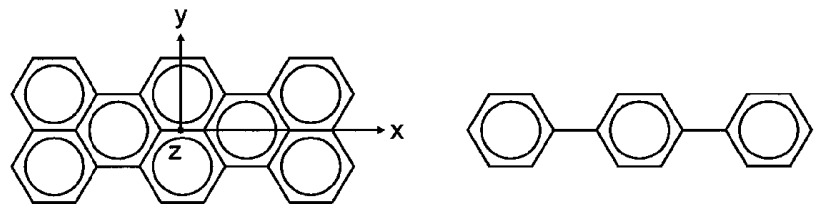

FIG. 1. The molecular structures of terrylene and $p$-terphenyl. 


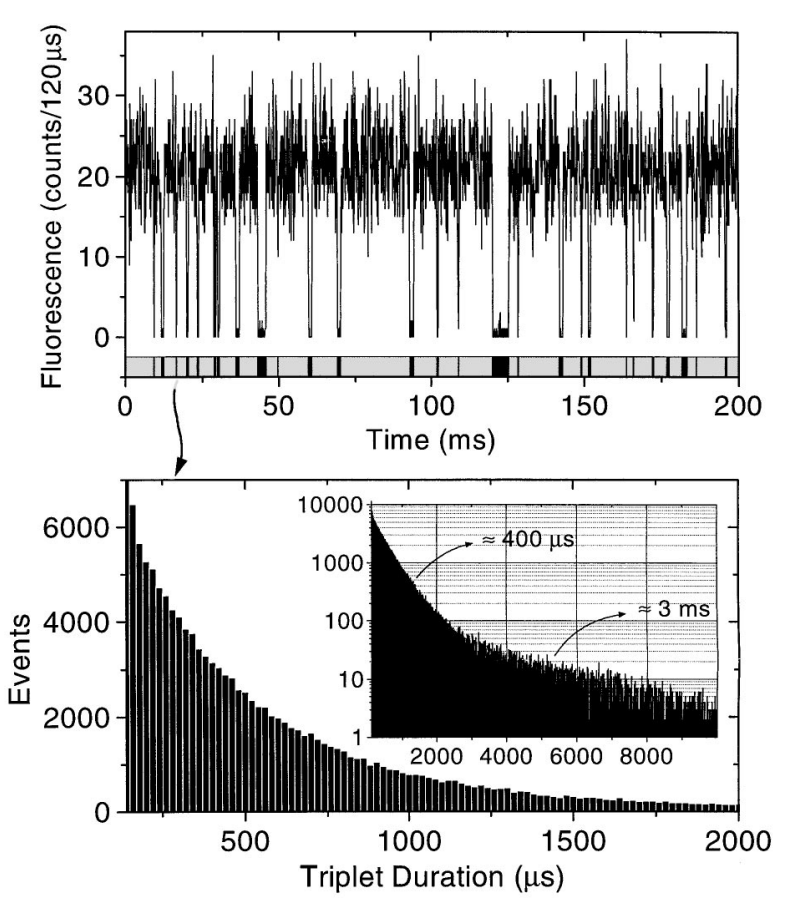

FIG. 2. Histogram of triplet durations for molecule 1 (bottom) and a part of the fluorescence signal from which it was obtained (top). The inset shows a semilog plot of the histogram which clearly indicates that there are at least two time constants in the triplet decay.

times of various durations at a range of microwave frequencies. Basically, at each microwave frequency a histogram as shown in Fig. 2 is measured. Clearly, at roughly $1276 \mathrm{MHz}$, the statistics change. Given that the long triplet-residence times disappear from the histogram, it must be that a transition between the long-lived and a short-lived sublevel is established. A zero-field triplet sublevel is labeled by the molecular axis $(x, y$, or $z$ as defined in Fig. 1) perpendicular to the plane in which the triplet spin is aligned. It will be shown later that the coupled long-lived and short-lived sublevels are $T_{z}$ and $T_{x}$, respectively.

To convert the histogram into a spectrum, a contrast measure that quantifies the degree to which the statistics change at a particular microwave frequency is required. The chosen measure is defined by

$$
\left(\sum_{i} \frac{\sum_{j} d(j)}{d^{\prime}(i)}\left[\frac{d^{\prime}(i)}{\sum_{j} d(j)}-\frac{h^{\prime}(i, \nu)}{\sum_{j} h(j, \nu)}\right]^{2}\right)^{1 / 2},
$$

where $d$ is the default triplet duration histogram measured without microwaves, $h$ is the $2 \mathrm{D}$ histogram, and $i$ and $j$ sum over the triplet durations. The prime indicates a duration dependent smoothing that compensates for the quantization noise. Since each contrast value is normalized to the number of detected triplet residences, this measure is insensitive to variations in the detected fluorescence rate induced by, e.g., mechanical drift or

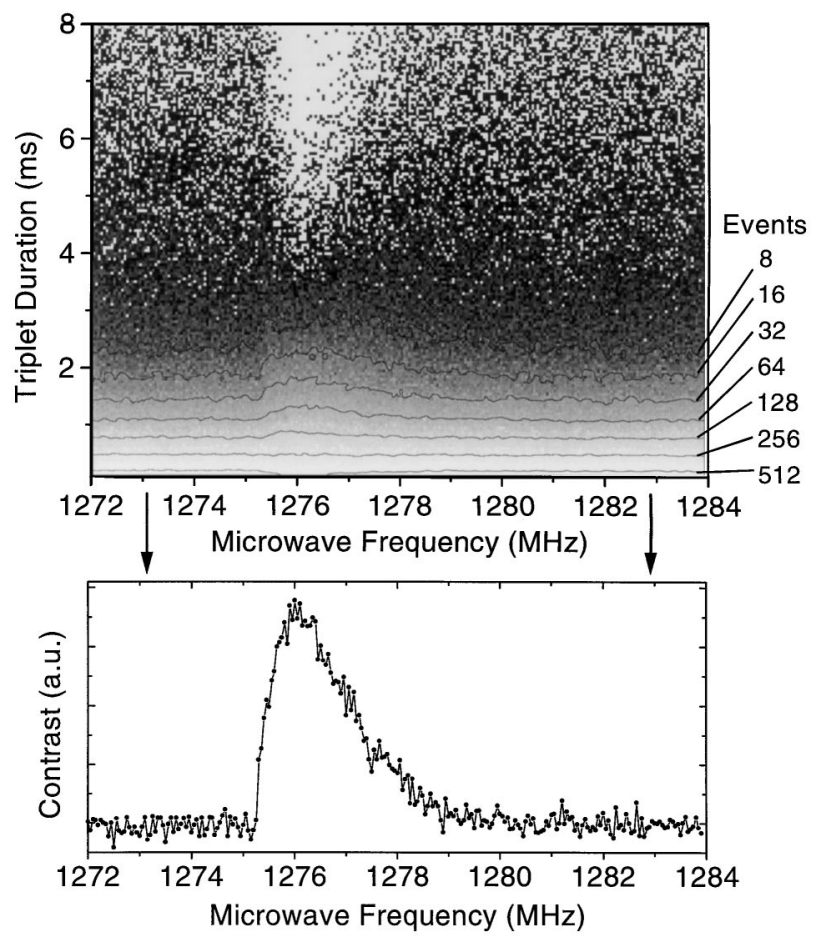

FIG. 3. Histogram of triplet duration versus microwave frequency for the $T_{x}-T_{z}$ transition of terrylene molecule 2 selected at $17286.25 \mathrm{~cm}^{-1}$. At each microwave frequency a histogram like that in Fig. 2 is plotted along the vertical axis. The grey scale and contours indicate the number of tripletresidence events at a frequency with identical duration. Isolated black pixels represent single events. The extracted spectrum is shown at the bottom.

changes in the intensity, polarization, or frequency of the exciting laser light.

In similar fashion, the transition between the long-lived and the other short-lived sublevel $\left(T_{y}\right)$ was detected at $1049 \mathrm{MHz}$ as shown in Fig. 4. Since the short-lived sublevels are close in energy for planar aromatic molecules, the transition frequencies give the fine-structure splitting of the triplet state of terrylene up to a mutual sign change; $E_{x}= \pm 501 \mathrm{MHz}, E_{y}= \pm 274 \mathrm{MHz}$, and $E_{z}=$ $\mp 775 \mathrm{MHz}$.

The asymmetric shape of the spectra in Figs. 3 and 4 is a consequence of the hyperfine interaction between the triplet electron spin and the nuclear spins in the

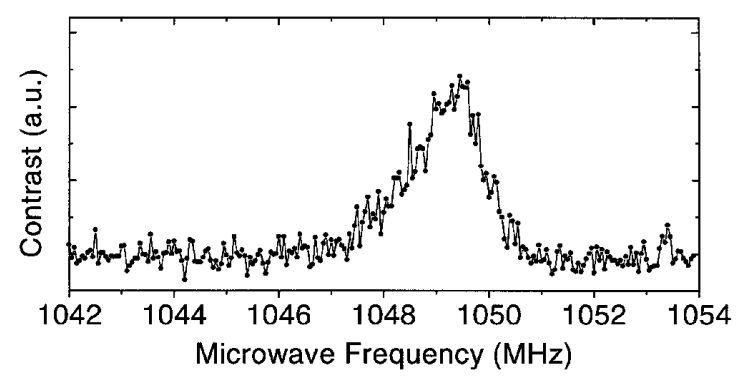

FIG. 4. $T_{y}-T_{z}$ transition of terrylene molecule 2. 
molecule [2]. For a fully ${ }^{12} \mathrm{C}$ terrylene molecule, only the protons bound to the molecule contribute since ${ }^{12} \mathrm{C}$ has no spin. However, ${ }^{13} \mathrm{C}$, a spin $I=1 / 2$ nucleus, has a natural abundance of $1.1 \%$. Thus, infrequently, molecules containing one or two ${ }^{13} \mathrm{C}$ nuclei may be found and these nuclei make a considerable contribution to the hyperfine interaction because the triplet spin density is mainly on the carbons. Figure 5 shows the spectrum of such a molecule; the line shape is indeed broadened by a few $\mathrm{MHz}$ relative to that in Fig. 3. The molecule was selected in the blue wing of the origin of the $X_{2}$ site which is consistent with the fact that an isotopic isomer containing ${ }^{13} \mathrm{C}$ experiences a slight blueshift of the optical transition. Analogous observations were made and quantified for pentacene [10]. Unfortunately, the spin density distribution for terrylene is unknown, which prohibits a quantitative analysis of the line broadening.

Additional experiments become possible once the frequencies of the zero-field transitions are known. In particular, the full kinetics of the triplet state can be determined. The histogram of triplet-residence times contains information on the lifetimes and on the relative yields of the triplet sublevels upon intersystem crossing. However, since the two short lifetimes are similar, these cannot be determined accurately from a three-exponential fit. By applying resonant microwaves, the molecule is quickly transferred between the coupled levels resulting in an effective decay rate that is the average of the rates for the individual levels. A histogram of triplet-residence times recorded while coupling sublevels exhibits a simplified decay behavior. To determine the kinetics, a simultaneous fit to three histograms was performed, one recorded without microwaves and the other two recorded while saturating the $T_{x}-T_{z}$ and $T_{y}-T_{z}$ transition, respectively. The corresponding functions used in the fit were

$$
\begin{gathered}
A_{1}\left[f_{x} k_{x} e^{-k_{x} t}+f_{y} k_{y} e^{-k_{y} t}+f_{z} k_{z} e^{-k_{z} t}\right], \\
A_{2}\left[\left(f_{x}+f_{z}\right) \frac{1}{2}\left(k_{x}+k_{z}\right) e^{-1 / 2\left(k_{x}+k_{z}\right) t}+f_{y} k_{y} e^{-k_{y} t}\right], \\
A_{3}\left[\left(f_{y}+f_{z}\right) \frac{1}{2}\left(k_{y}+k_{z}\right) e^{-1 / 2\left(k_{y}+k_{z}\right) t}+f_{x} k_{x} e^{-k_{x} t}\right],
\end{gathered}
$$

with $k_{x}, k_{y}$, and $k_{z}$ the sublevel decay rates and $f_{x}, f_{y}$, and $f_{z}$ the sublevel yield fractions (constrained to sum to

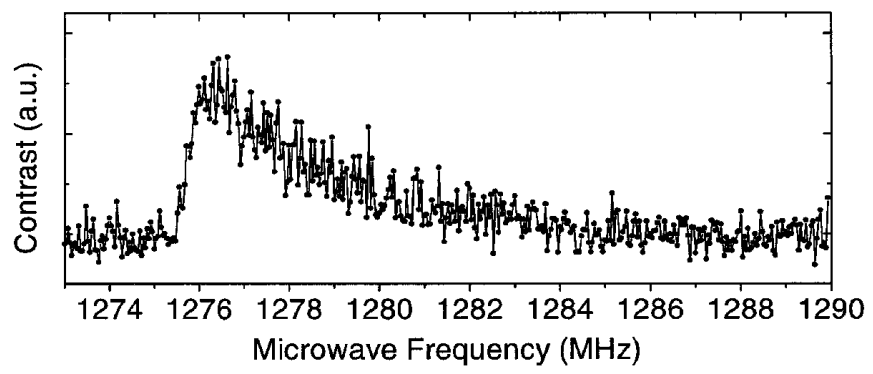

FIG. 5. $T_{x}-T_{z}$ transition of terrylene molecule 3 selected at $17287.80 \mathrm{~cm}^{-1}$. The broadened line shape indicates that the molecule contains ${ }^{13} \mathrm{C}$. one). The scaling parameters $A_{1}, A_{2}$, and $A_{3}$ absorb the somewhat different accumulation times of the histograms.

An interesting question is whether it is possible to determine the absolute ISC rates from $S_{1}$ to the $T_{1}$ sublevels. Vogel et al. [8] analyzed the duration of light periods just prior to long and short dark periods so as to determine the ISC rates to the long- and short-lived triplet sublevels. In contrast to their results we do not find any dependence of the light period statistics on the length of the subsequent dark periods. Indeed, from a conventional kinetic scheme with constant rates no such dependence is predicted; when in the singlet manifold, the molecule does not "know" in what triplet sublevel it is going to end up. Instead, the total intersystem crossing rate from $S_{1}$ to $T_{1}$ can be determined by recording a histogram of the singlet-residence times while optically saturating the $S_{0}-S_{1}$ transition. Under these conditions, the molecule has a probability of onehalf to occupy either the $S_{0}$ or $S_{1}$ state while it resides in the singlet manifold. Thus the (single exponential) decay rate observed in the singlet-residence time histogram equals half the intersystem crossing rate. The rate for an individual sublevel can be obtained by multiplying the total rate by the sublevel yield fraction. The results are summarized in Table I. Note that the change in average fluorescence under $\mathrm{cw}$ microwave excitation predicted from these numbers is, under ideal conditions, less than $1 \%$ for all three transitions. Detecting these transitions using classical ODMR would have been difficult particularly since the on/off switching of the fluorescence on a millisecond time scale necessitates long averaging times. Other molecules belonging to the $X_{2}$ site were observed to have similar rates and yields but the results probably do not generalize to terrylene molecules in other sites.

The experiments described above were performed by passively recording the quantum jumps. It is also possible to change the excitation parameters in reaction to a change of state of the molecule. In particular, one can trigger microwave pulses as soon as the molecule enters the triplet state. To minimize the response time, a simple electronics for generating a trigger upon a drop in the photon-counting rate was implemented. By accumulating the triplet-residence times as a function of the pulse width with the microwaves tuned in resonance with one of the zero-field transitions, a transient-nutation signal can be observed. Figure 6 shows the resulting 2D histogram for the $T_{x}-T_{z}$ transition together with the extracted

TABLE I. Kinetic parameters of terrylene molecule 1 selected from the $X_{2}$ site.

\begin{tabular}{cccr}
\hline \hline$T_{1}$ sublevel & $\begin{array}{c}\text { Decay rate } \\
\left(\mathrm{s}^{-1}\right)\end{array}$ & $\begin{array}{c}\text { Lifetime } \\
(\mu \mathrm{s})\end{array}$ & $\begin{array}{c}\text { Yield } \\
(\%)\end{array}$ \\
\hline$T_{x}$ & $2961 \pm 28$ & $338 \pm 3$ & $59.3 \pm 0.5$ \\
$T_{y}$ & $1761 \pm 25$ & $568 \pm 8$ & $33.6 \pm 0.6$ \\
$T_{z}$ & $317 \pm 9$ & $3153 \pm 86$ & $7.1 \pm 0.2$ \\
& $S_{1} \rightarrow T_{1}$ ISC rate $=540 \pm 17 \mathrm{~s}^{-1}$ \\
\hline \hline
\end{tabular}




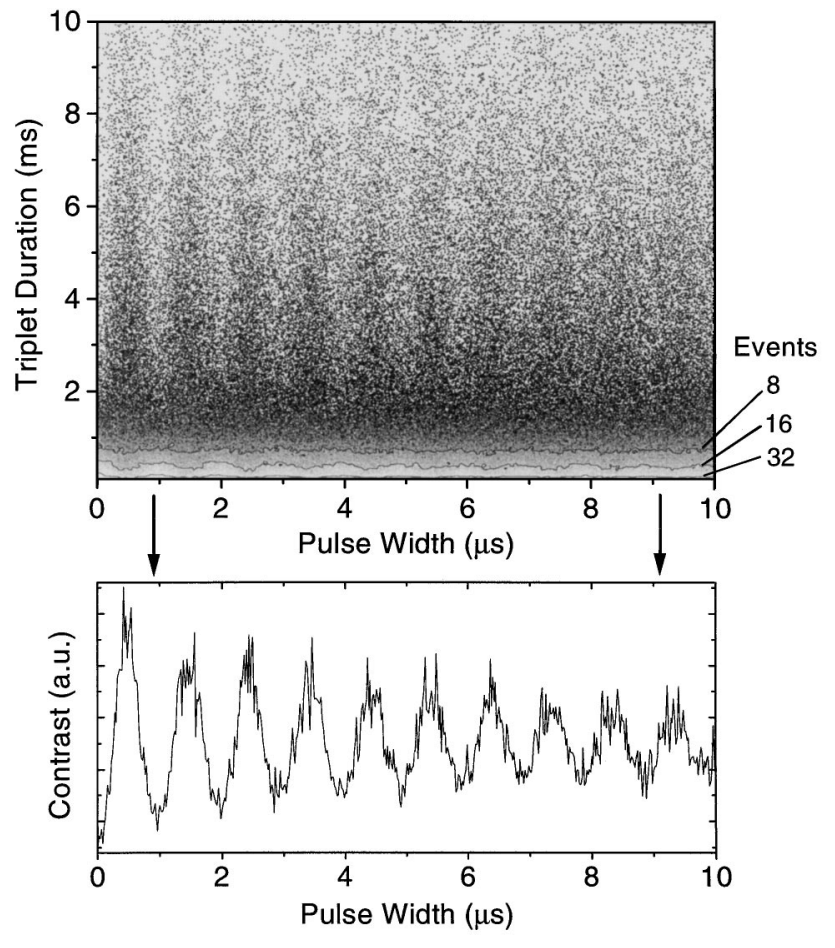

FIG. 6. Histogram of triplet duration versus microwave pulse width for the $T_{x}-T_{z}$ transition of molecule 1 together with the resultant transient-nutation trace. The Rabi frequency is $1.01 \mathrm{MHz}$.

contrast trace. Clearly, the molecule makes Rabi flops between the short- and long-lived sublevel. Analogous optically detected spin coherence has been observed for single pentacene molecules by Wrachtrup et al. [11]. In Fig. 7 the $T_{y}-T_{z}$ transient-nutation signal is shown. It was recorded under identical microwave power, yet the Rabi frequency differs markedly. The Rabi frequency for a transition is proportional to the projection of the microwave $\vec{B}$ field on the direction of polarization of the transition between the coupled sublevels. The microwave field was aligned perpendicular to the $p$-terphenyl crystal plane, and the crystal structure is such that the long axes of the $p$-terphenyl molecules make an angle of $17^{\circ}$ with

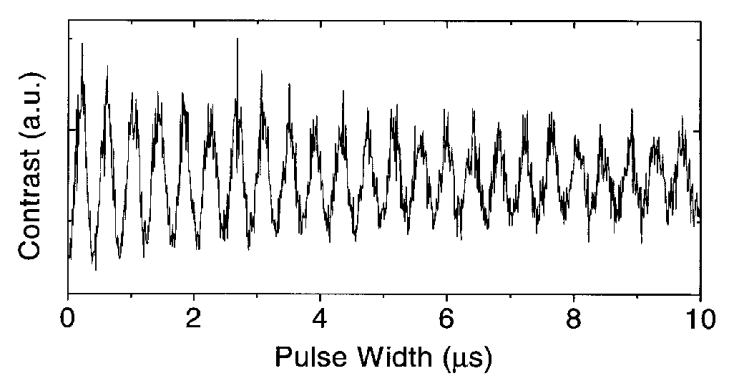

FIG. 7. Transient-nutation trace for the $T_{y}-T_{z}$ transition of molecule 1. The Rabi frequency is $2.41 \mathrm{MHz}$. the crystal plane normal [12]. Given the structure of the molecules (Fig. 1), it is reasonable to assume that the long axis of the terrylene guest molecule is aligned with the long axes of the $p$-terphenyl molecules making up the host matrix. This assumption is corroborated by the assignment of the insertion sites of terrylene in $p$-terphenyl by Kummer et al. [13]. Thus the highest Rabi frequency is expected for the $T_{y}-T_{z}$ transition. This information, together with the fact that for the long-lived level the spin is aligned in plane, allowed us to associate the triplet sublevels with particular molecular axes.

In conclusion, the outlined technique offers a direct way of detecting magnetic-resonance transitions for single molecules with a very low degree of intersystem crossing. The method relies on the analysis of the statistics of quantum jumps between the singlet and triplet manifold. As demonstrated for terrylene it opens the possibility of studying systems inaccessible to conventional optically detected magnetic resonance.

This work forms part of the research program of the "Stichting voor Fundamenteel Onderzoek der Materie" (FOM) and is financially supported by the "Nederlandse Organisatie voor Wetenschappelijk Onderzoek" (NWO).

[1] Single-Molecule Optical Detection, Imaging and Spectroscopy, edited by T. Basché, W. E. Moerner, M. Orrit, and U.P. Wild (VCH, Weinheim, 1997).

[2] J. Köhler, J. A. J. M. Disselhorst, M. C. J. M. Donckers, E. J. J. Groenen, J. Schmidt, and W. E. Moerner, Nature (London) 363, 242 (1993).

[3] J. Wrachtrup, C. von Borczyskowski, J. Bernard, M. Orrit, and R. Brown, Nature (London) 363, 244 (1993).

[4] A. Gruber, A. Dräbenstedt, C. Tietz, L. Fleury, J. Wrachtrup, and C. von Borczyskowski, Science 276, 2012 (1997).

[5] S. Kummer, T. Basché, and C. Bräuchle, Chem. Phys. Lett. 232, 414 (1995).

[6] T. Plakhotnik, W. E. Moerner, T. Irngartinger, and U.P. Wild, Chimia 48, 31 (1994).

[7] T. Basché, S. Kummer, and C. Bräuchle, Nature (London) 373, 132 (1995).

[8] M. Vogel, A. Gruber, J. Wrachtrup, and C. von Borczyskowski, J. Phys. Chem. 99, 14915 (1995).

[9] M. Kinoshita and N. Iwasaki, Appl. Spectrosc. Rev. 17, 1 (1981).

[10] A.C.J. Brouwer, J. Köhler, E.J.J. Groenen, and J. Schmidt, J. Chem. Phys. 105, 2212 (1996).

[11] J. Wrachtrup, C. von Borczyskowski, J. Bernard, M. Orrit, and R. Brown, Phys. Rev. Lett. 71, 3565 (1993).

[12] J. L. Baudour, Y. Delugeard, and H. Cailleau, Acta Crystallogr. Sect. B 32, 150 (1976).

[13] S. Kummer, F. Kulzer, R. Kettner, T. Basché, C. Tietz, C. Glowatz, and C. Kryschi, J. Chem. Phys. 107, 7673 (1997). 